\title{
LIVER EXTRACTION USING HISTOGRAM AND MORPHOLOGY
}

\author{
S. Kiruthika ${ }^{1}$, I. Kaspar Raj ${ }^{2}$ \\ ${ }^{1}$ Women Scientist, Computer Centre, Gandhigram Rural Institute-Deemed University, Tamilnadu \\ 1'kiruthikasm09@gmail.com \\ ${ }^{2}$ Director i/c, Computer Centre, Gandhigram Rural Institute-Deemed University, Tamilnadu \\ ${ }^{2}$ kasparraj@gmail.com
}

\begin{abstract}
Liver is the largest glandular organ important for survival in human body. Computed tomography is generally used to image liver due to its precision. This paper presents a method to extract liver from computed tomography (CT) abdomen images in axial orientation. A traditional segmentation method based on histogram and morphology is proposed herein. Histogram is used to analyze the intensity distribution, morphological operations are used to disconnect liver from the neighboring organs and greatest connected pixels are extracted. The experimental results of the proposed method when applied to CT abdomen images with contrast are presented and the effectiveness is discussed in accordance to the manual tracing obtained from the radiologist. Dice similarity co-efficient amounts to $94 \%$ in the proposed method.
\end{abstract}

Key Words: segmentation, extraction, histogram, morphology, connected component, ct liver

\section{INTRODUCTION}

Liver is one of the most important organs in the human body. It carries out varieties of functions such as filtering the blood, making bile and proteins, processing sugar, hormone production, breaking down medications and storing iron, vitamins and minerals[1]. Liver weights approximately $1500 \mathrm{~g}$, and is located in the upper right corner of the abdomen. Liver is the largest organ in the abdominal cavity. Liver disease is one of the most serious health diseases that cause death worldwide [2]. In modern medicine, medical imaging techniques has major role in helping diagnosis. Medical imaging is the technique and process of creating visual representation of the function of some organs or tissues. Various types of imaging technologies based on non-invasive approach are X-rays, Computed Tomography (CT) scan, Magnetic Resonance Imaging (MRI) scan, ultrasound, Positron Emission Tomography (PET) etc. These imaging technologies are used to view the human body in order to diagnose, monitor, or treat medical conditions. Each type of technology gives different information about the body being studied or treated. Liver image segmentation has played a very important role in medical imaging field. The advancement in digital image processing techniques has attracted researchers towards the development of computerized methods for liver analysis.

Liver image segmentation is an essential step for the diagnosis of liver tumors, liver surgical planning system such as a system for liver transplantation and 3D liver volume rendering. Computed Tomography image has been widely used for liver disease diagnosis. Computed Tomography images can be taken either with contrast agent given to the patients or without contrast agent according to the necessity. The most challenging task in segmenting liver from the CT abdomen image is highly varying shape of the liver, weak boundaries to its adjacent organs such as stomach, kidney, and heart and the intensity homogeneity between adjacent organs [3]. The axial section of computed tomography (CT) of the abdomen image is shown in Fig-1.

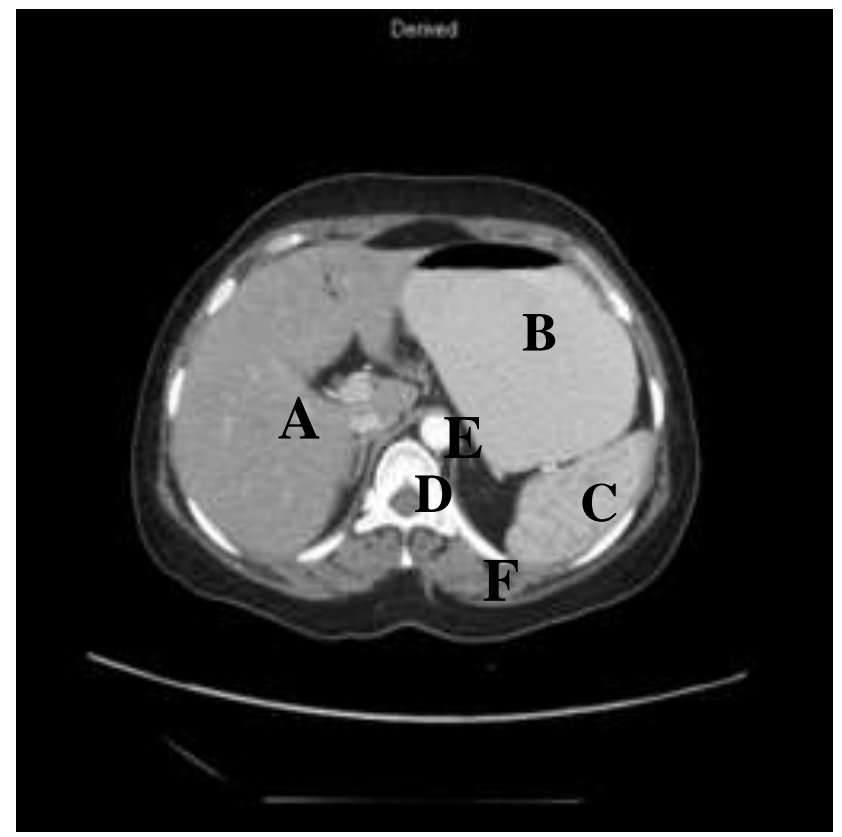

Fig-1: CT scan of Abdomen Image $\left(\mathrm{I}_{\mathrm{R}}\right)(\mathrm{A})$ Liver; (B)Stomach; (C)Spleen; (D)Spine; (E) Aorta; (F) Fat

Prior knowledge about location of the organ and image features is required before segmenting liver from CT abdomen images. The extraction of liver region from CT abdomen images using histogram analysis and morphological operations is proposed in this paper.

This paper is organized as follows: various approaches used for liver segmentation is reviewed in section 2. Materials used in this method are given in section 3. Section 4 
describes the image processing methods of the proposed technique for liver extraction. Experimental results of the proposed method are discussed in section 5. Conclusion is given in Section 6.

\section{PREVIOUS WORKS IN THE LITERATURE}

An automatic segmentation method based on graph cuts [4] separates the images into two classes "object" and the "bottom" separates liver from CT images. Semi automatic method based on 2D region growing with knowledge based constraints proposed in [5]. Neural network classifier [6] is used to obtain an initial estimation of the liver region. Liver segmentation with region growing is proposed in [7]. The seed point required for region growing is automatically found by taking the centroid of the biggest connected area in the simplified and eroded image. In [8] automatic liver segmentation of contrast enhanced CT images is carried out.

\section{MATERIALS AND METHODS}

In this method, two dimensional Computed Tomography axial abdomen images with contrast agents are used. The contrast agents may be given to the patient in either of the two ways: (1) oral, (2) intravenous [9]. These contrast agents causes the particular organ or tissue under study to be viewed better. These images are collected from Medall Diagnostics. The dataset volume has 35 slices, of which only 20 slices will have liver region.

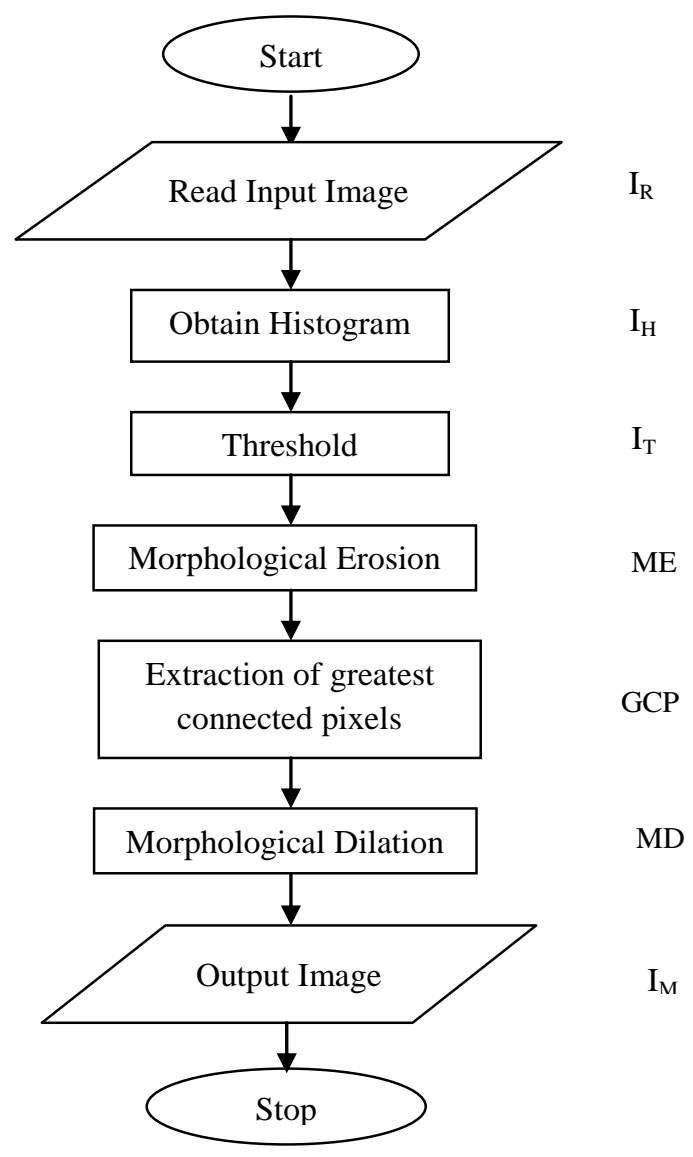

Fig-3: Flow chart of the proposed method
The proposed method uses histogram, thresholding and morphological segmentation for extracting liver region from CT abdomen images.

\section{PROPOSED METHOD}

The principle of the proposed method is given in Fig-3. Generally, the threshold value is used to separate liver, which is the region of interest (ROI) from the background image.

But in our case, according to the histogram of the image slices of the abdominal CT, it is found that histogram [10] of the liver has a shape as shown in Fig-2 with peak values that lies between $\mathrm{t} 1$ and $\mathrm{t} 2$.

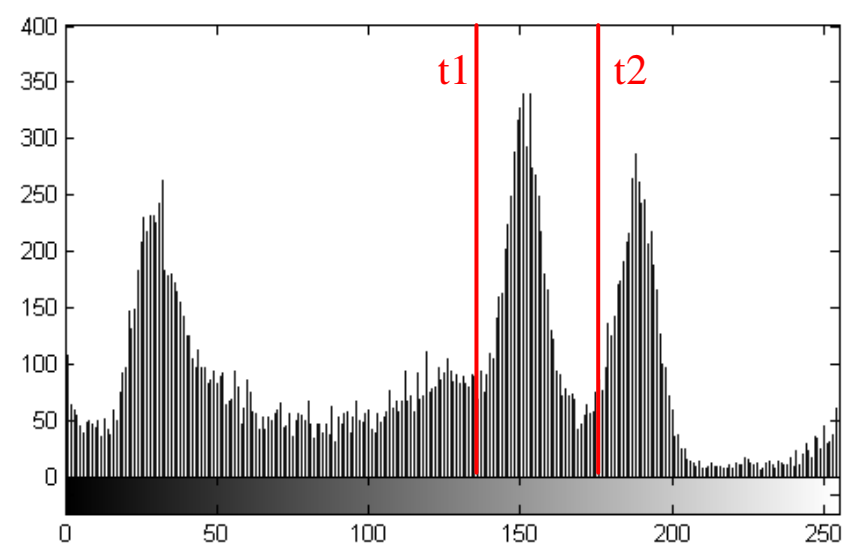

Fig-2: Histogram of fig.1 $\left(\mathrm{I}_{\mathrm{H}}\right)$

\subsection{Thresholding}

With the values $t 1$ and $t 2$, the threshold is computed. For the first threshold, $\mathrm{t} 1$ eliminates all the parts of the histogram to the left of $\mathrm{t} 1$ and for the second threshold, the parts that remains to the right of $\mathrm{t} 2$ is removed. The left over portion of the image is the pixels that exist in the interval $\mathrm{t} 1$ and $\mathrm{t} 2$. The image is then converted into a binary image by taking threshold $\left(\mathrm{I}_{\mathrm{T}}\right)$. The binary image consists of liver, spleen and fat muscles that surround the abdomen which has similar intensity levels.

\subsection{Morphological Erosion}

The binary image obtained in the previous step contains other organs which have similar intensity to liver and they are also connected to liver. To remove the continuities between them and to separate liver from the abdomen boundary, morphological erosion (ME) operation is implemented. Fig.4 (a) and (b) shows the original image and the resultant image of erosion respectively. 


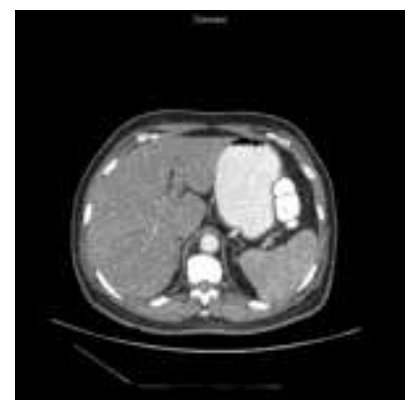

a

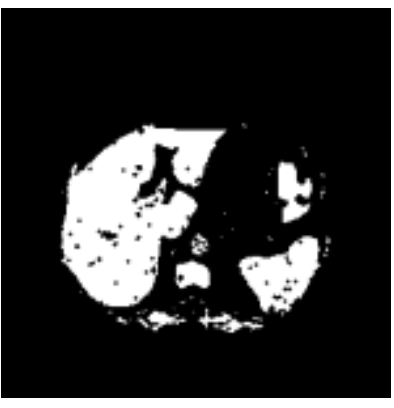

b
Fig-4: a) Original Image; b)Eroded Image after thresholding

\begin{tabular}{|l|l|l|l|l|}
\hline 1 & 1 & 1 & 1 & 1 \\
\hline 1 & 1 & 1 & 1 & 1 \\
\hline 1 & 1 & 1 & 1 & 1 \\
\hline 1 & 1 & 1 & 1 & 1 \\
\hline 1 & 1 & 1 & 1 & 1 \\
\hline
\end{tabular}

Fig-5: Structuring element (SE3)

Erosion [11] is an operation which removes the pixels from the object boundaries. A structuring element is a matrix consisting of only 0 's and 1's with any arbitrary shape. In this method disk shaped structuring element is used. The disk shaped structuring element of radius 3 as shown in Fig5 is used in this method. The erosion process is shown in eq. 1

$\mathrm{ME}=\mathrm{I}_{\mathrm{T}} \Theta \mathrm{SE} 3$

\subsection{Greatest Connected Pixels}

Once the image is eroded, the abdomen boundaries are removed. Also the continuities between liver and its neighboring organs are disconnected. Since liver is the largest connected organ in the left side of CT abdomen images, taking out the greatest connected pixels extract the liver region exactly. The greatest connected pixels are detected with labeling process. The pixels which share similar intensities are grouped under a certain unique label. Finally the label with greatest quantity is extracted, which is liver.

\subsection{Morphological Dilation}

The extracted image (GCP) is then dilated with disk operator of radius 3 (SE3), for regaining the necessary elements which were lost during erosion. Dilation and erosion are dual operators. Dilation [11] thickens object in the binary image. Dilation is generally used for bridging gaps. The dilation process is shown in eq. 2

\section{$\mathrm{MD}=\mathrm{GCP} \bigoplus \mathrm{SE} 3$}

The resultant image is a binary liver mask $\left(\mathrm{I}_{\mathrm{M}}\right)$ which is then overlapped with the original image to obtain the liver region.

\section{RESULT AND DISCUSSION}

Fig-6 shows the raw image dataset of CT abdomen and Fig7 shows the segmentation result produced by the proposed procedure.

In the dataset shown in the Fig-6, first 4 slices contain liver, aorta, spine and heart inside the abdominal wall, in the next 8 slices liver, stomach, aorta, spine, and spleen can be seen and in the remaining 7 slices liver, aorta, left and right kidneys, stomach, spleen, and pancreas are visible. Liver is the organ which is located in the left side (which is actually in the right side of the human body) of the CT axial image is largest organ inside the abdomen.

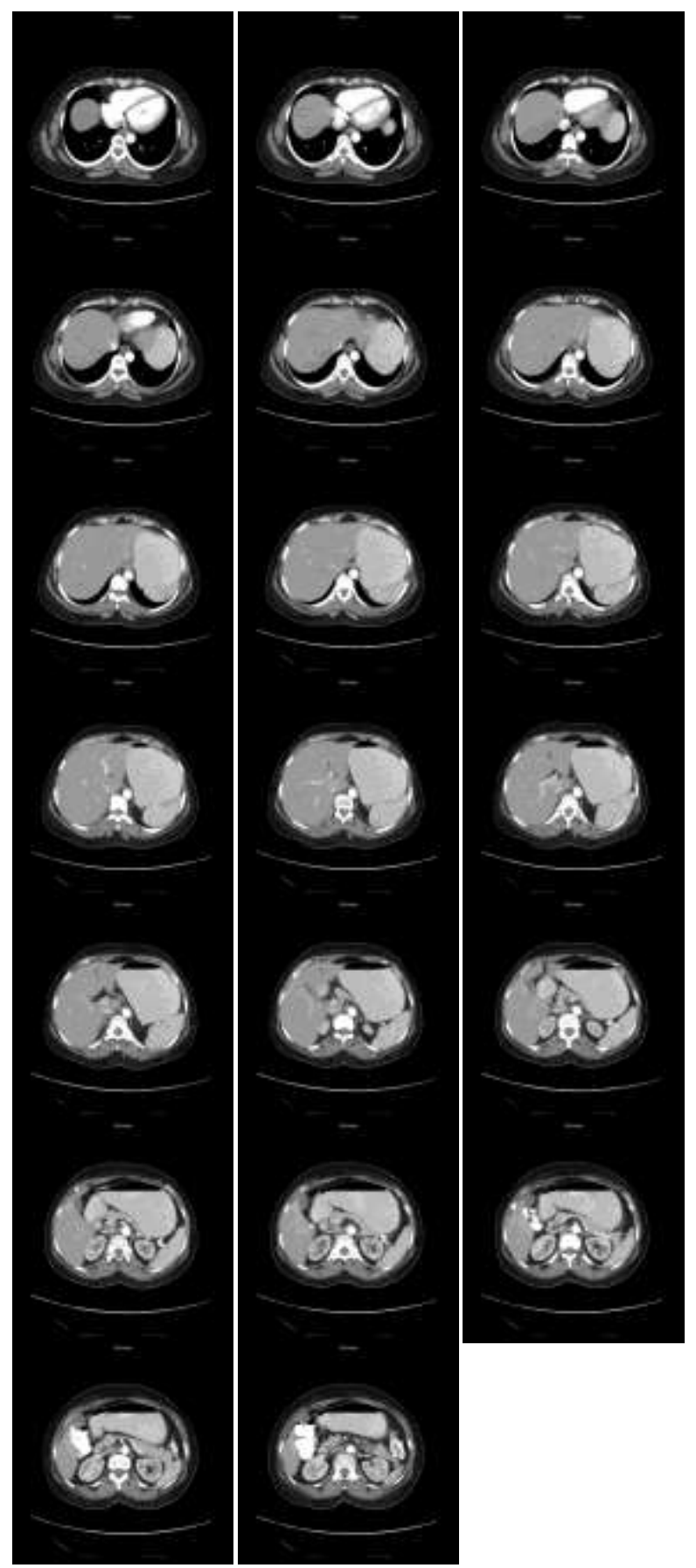

Fig-6: Raw images of Computed Tomography 


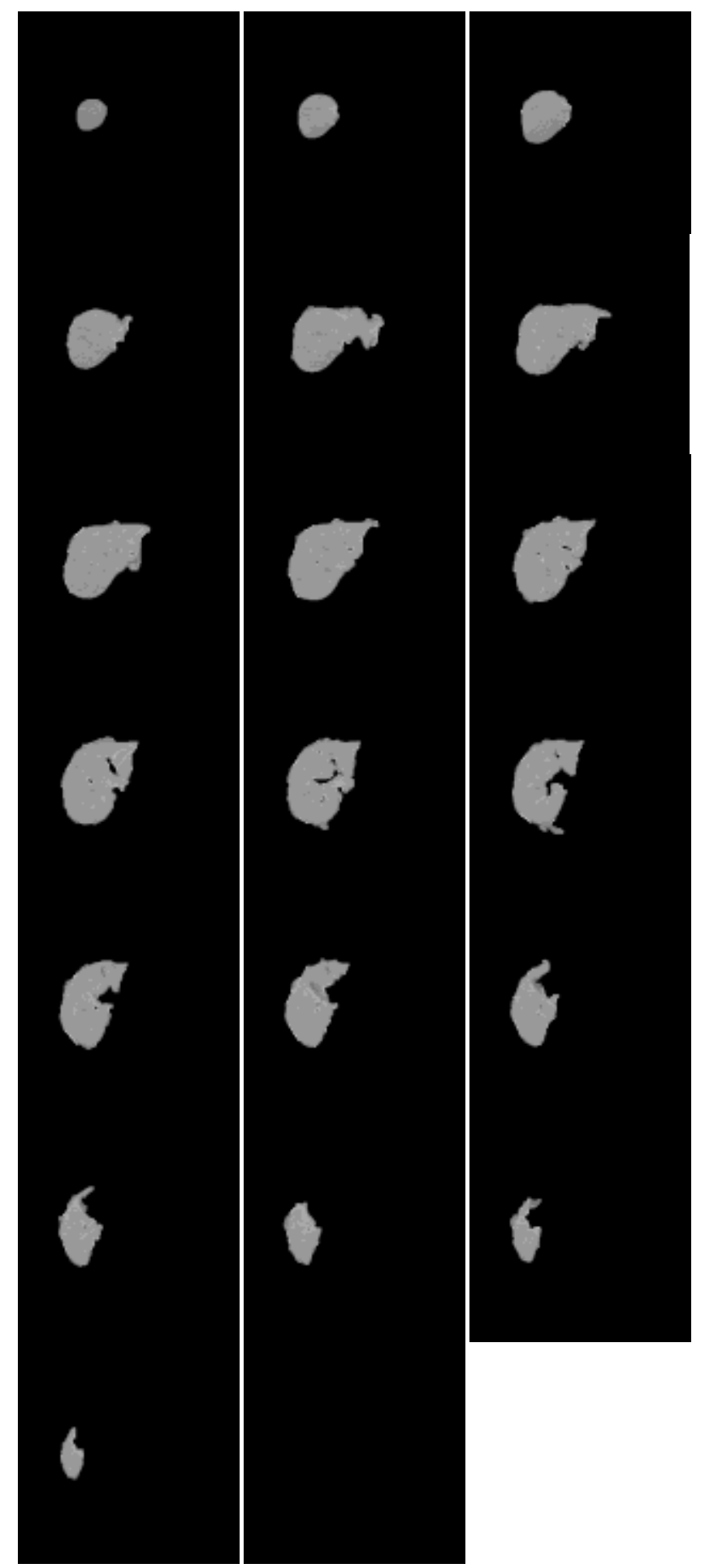

Fig-7: Liver region segmented images

Experiments are conducted on a 2D CT axial abdomen images with contrast. The average number of slices per case was 35 , and the slice interval in $1 \mathrm{~mm}$. Organs focused in this scan were heart, liver, stomach, kidneys, spleen, aorta, inferior vena cava, pancreas and gall bladder. From this image, the proposed method segments liver region.

The results obtained from the proposed method are validated with manual segmentation obtained from the radiological expert. The Dice co-efficient [12] is the most used metric for validating the results of segmentation based on similarity. We justify the proposed segmentation method by the measure the agreement between proposed and manual segmentation. Dice values are expressed in percentage and obtained using eq. 3

Dice $=\frac{2|A \cap B|}{2|A \cap B|} * 100$

Here, A is the proposed method and B is the manual segmentation obtained from radiologist. The average value of the dice co-efficient for this volume is $94 \%$.

In this execution, the result obtained by the proposed method need refinement in the area where the joins exists between abdominal wall and liver also between fat muscles and liver. The proposed method shows average results for CT abdomen-contrast images. To achieve commendable result, the method should be meticulously refined with the dominating knowledge about image and advanced segmentation techniques.

\section{CONCLUSION}

A liver segmentation method is proposed using histogram analysis and morphological operations for extracting liver from the $\mathrm{CT}$ abdomen images. This method inputs raw ct abdomen images. By finding the threshold interval of the liver region with the histogram prediction, gray scale image is converted into a binary image. Morphological erosion is used to erode the pixels that do not come under ROI. Extracting the greatest connected pixels, results in liver extraction. Dilation brings back the lost pixels in the ROI. Selecting the shape of structuring element plays an important role in implementing morphological operations. Overlapping the mask obtained in the previous result to the raw image pull out the liver region. Dice similarity coefficient is used to compare the results of the proposed method with manual segmentation, which results to $94 \%$. The future work will evolve with the feature to separate the boundaries of liver with the neighbors and from the abdominal wall.

\section{ACKNOWLEDGEMENT}

This research work is assisted by Department of Science and Technology, Govt. of India under women scientist scheme. We would like to thank Dr. Vidyasagar, M.B.B.S., M.D(Rad), consultant radiologist, Medall Diagnostics for providing real datasets with manual segmentation.

\section{REFERENCE}

[1] Maton, et al.: Human Biology and Health. Englewood Cliffs, New Jersey, USA, 1993.

[2] Ferlay J, Soerjomataram I, Ervik M, Dikshit R, Eser S, Mathers C, Rebelo M, Parkin DM, Forman D, Bray F.: Cancer Incidence and Mortality Worldwide: IARC CancerBase No. 11, GLOBOCAN 2012.

[3] Shraddha Sangeswar, Dr.Prema Daigavane,: Liver Segmentation of CT scan Images-A Survey, 
International journal on Recent and Innovation Trends in Computing and Communication, vol.3, 2015.

[4] M.Usman Akram, Aasia Khanum and Khalid Iqbal.: An automatic system for Liver CT Enhancement and Segmentation, ICGST,-GVIP Journal, vol.10, Issue IV, October 2010.

[5] Yingyi Qi,Wei xioong,Wee keng leow.: "Semiautomatic segmentation of liver tumors from CT scans using Bayesian rule based 3D region growing", Grand challenge, 2008.

[6] Gletsos, et al.: "A computer-aided diagnostic system to characterize CT focal liver lesions: Design and optimization of a neural network classifier," IEEE Tram. Inform. Tech" Biomed., in press, 2003.

[7] Kumar S. S., Moni R. S., Rajeesh J.: Automatic Segmentation of Liver and Tumor for CAD of Liver, Journal of advances in information technology, vol. 2, pp: 63-70, 2011.

[8] Rusko L, Bekes G., Nemeth G, \& Fidrich, M.: Fully automatic liver segmentation for Contrast enhanced CT images. MICCAI Workshop. 3D Segmentation in the Clinic: A Grand Challenge, 2(7), 2007.

[9] Hage Stephen John, Shratter, Lee Alan.: CT Scans, Gale Encyclopedia of surgery: A guide for parients and caregivers, Encyclopedia.com, 2004.

[10] Oussema Zayane, Besma Jouini, Mohamed Ali Mahjoub.: Automatic liver segmentation method in CT images, Canadian Journal on Image Processing \& Computer Vision, vol.2, No.8, December 2011.

[11] Rafael C. Gonzalez, Richard E. Woods.: Digital Image Processing, Second Edition. 2007.

[12]Dice, L.R: Measures of the amount of ecologic association between species. Ecology,26, 297-302 1945. 